\title{
Network Security Situation Analysis of Weighted Neural Network with Association Rules Mining
}

\author{
Jie DU ${ }^{1, a}$, Hongna LUO ${ }^{2, b}$ \\ ${ }^{1}$ PINGDINGSHA INSTITUTE OF EDUCATION, Ping Ding Shan city, HeNan, China \\ ${ }^{2}$ PINGDINGSHA INSTITUTE OF EDUCATION, Ping Ding Shan city, HeNan, China
} Keywords: Tristate Wireless Sensor Networks; Multistate and Multiple-Valued Decision Diagram;
Reliability Assessment; Transmission Delay of Data; Imperfect Cover; Common Cause Failure

\begin{abstract}
In order to assess reliability of polymorphic wireless sensor networks, specific to characteristics of communication delays, imperfect cover (IPC) and common cause failure (CCF) which are different from general network, a reliability evaluation model of multistate WSN is constructed, which simultaneously considers processing delay of node data, IPC and CCF. Specific to the problem of "combinatorial explosion" produced by system state space of multimode WSN with the increasing of node number, in order to avoid solving the minimal path set, multistate and multiple-valued decision diagram (MMDD) is introduced to build WSN as required. By just constructing one MMDD, according to common cause set, reliability of WSN can be calculated under the effect of IPC and CCF, which effectively controls the space complexity of the algorithm. Experimental results show that the provided MMDD algorithm can complete reliability assessment task of polymorphic WSN constrained by IPC、CCF and time delay. The calculated reliability value is lower than the result of solution method for model of WSN ignoring the network congestion and the influence of CCF and IPC.
\end{abstract}

\section{Introduction}

The development of micro electro mechanical systems and wireless technology recently has arouse the research upsurge of wireless sensor networks (Sensor Networks Wireless, WSN) in various application fields, of which the reliability evaluation is the key task before network deployment. Traditional WSN model of two node states underestimates reliability of network. Description of WSN node polymorphism is more in line with the characteristics of the sensor network ${ }^{[1-2]}$. In addition, WSN node construction, working environment and the inherent redundancy of the network itself and other attributes make WSN have the characteristics different from common network in aspects of communication delays, node failure and failure correlations etc, of which the problem of reliability is more complicated.

\section{Design of Weighting Network Stabilization}

\section{Polymorphic WSN model and reliability under constraint of time delay}

WSN model of node tristate, namely, $3 s-W S N=<V, E, M, Q, S, v_{\text {sink }}>, V$ is the set of sensor node in 3s-WSN; $E=\left\{e=\left(v^{\prime}, v\right) \mid v^{\prime}, v \in V\right\}$ is the physical link set among sensor nodes; $M=\left\{m_{\mathrm{s}}, m_{\mathrm{co}}, m_{\mathrm{b}}\right.$, $\left.m_{0}\right\}$ is function module set of sensor node; $m_{s}$ is the sensing module; $m_{c o}$ is the wireless module; $m_{b}$ is the data cache module; $m_{o}$ is other module. It is agreed that only MS and MCO may have a failure with two states of reliability and failure. $Q=\left\{q_{\mathrm{s}}, q_{\mathrm{co}}, q_{\mathrm{b}}, q_{\mathrm{o}}\right\}$, failure probability of each function module of sensor node, $q_{\mathrm{b}}, q_{\mathrm{o}}=0, q_{\mathrm{s}}, q_{\mathrm{co}} \in(0,1) ; S$ is sensor node state space; $v_{\text {sink }}$ is the sink node, which is responsible for communication with end users in the current network. From the achievable functional features of nodes, three node states will appear after combination of running state of each function module, namely $S=\{c s, c, f\}$ : $c s$ state, all modules are working properly; nodes can collect data and can also complete the data transmission; $c$ state, only MS has failure and the node cannot collect data but it can accept data collected by other nodes; $f$ state, $m_{c o}$ has failure, nodes cannot achieve data transmission in complete failure. For any node $v \in V$, The probability of 
each state is denoted as $p_{c s}(v), p_{c}(v)$ and $p_{f}(v)$.

WSN node's ability to transmit data is limited. Time delay from end to end communication not only includes the transmission delay of link consumption, but also the queuing delay caused by data flow blocking in node buffer must be considered. Assumed that $g(v)$ is the data volume generated in unit time of $m_{s}$ module of node $v ; \beta(v)$ is the data volume transmitted in unit time of $m_{c o}$ module of node $v ; \theta(v)$ is the data volume transmitted by upstream node of node $v$ in unit time. Assumed that:

$$
\theta(v)=\sum_{\left(v^{\prime}, v\right) \in E} \frac{p_{c s}\left(v^{\prime}\right) \cdot\left[g\left(v^{\prime}\right)+\theta\left(v^{\prime}\right)\right]+p_{c}\left(v^{\prime}\right) \cdot \theta\left(v^{\prime}\right)}{\text { outDegree }\left(v^{\prime}\right)}
$$

Where, outDegree $\left(v^{\prime}\right)$ is the number of upstream node of node $v^{\prime} ; \lambda(v)$ is the data volume in module $m_{b}$ of the node $v$ in unit time. When $v$ is in the state of $c s, \lambda(v)$ includes $g(v)$ and $\theta(v)$; when $v$ is in the state of $c, \lambda(v)$ is $\theta(v)$.

For any node $v \in V$, data volume to be transmitted is recorded as $W_{v}(t)$ in data cache module $m_{b}$ of node $v$ at the time of $t$, and

$$
W_{v}(t)=\sum_{i=1}^{t}\left[\lambda(v)+W_{v}^{*}(t-1)\right]
$$

Where, $t=1,2,3, \ldots ; W_{v}{ }^{*}(t-1)$ is the data volume failing to be forwarded by data cache module $m_{b}$ of node $v$ at the time of $(t-1)$. If the initial state of $v$ is in the state of $c s, W_{v}(0)=g(v)$; if the initial state of $v$ is in the $c$ state, $W_{v}(0)=0$.

Assumed that tolerable upper limit of time delay of end users is $T \in \Phi^{+}$, there is a link from the source node $v_{0} \in V$ to $v_{\text {sink.. }}$ When and only if the $v_{0}$ can generate data in time $\mathrm{T}$ and successfully transfer it to $v_{\text {sink }}$, rPath $v^{v_{0} \rightarrow v_{\text {sink }}}$ is reliable namely $\sum_{i=1}^{n}$ delay $_{v_{i-1} \rightarrow v_{i}}\left(t_{i-1}\right) \leq T . t_{i-1}$ is the consumed time of generating data and successfully transmitting it to $v_{i-1}$ of $v_{0}$. delay $v_{v_{i-1} \rightarrow v_{i}}\left(t_{i-1}\right)$ is the consumed time of data generated by $v_{0}$ at the time of $t_{i-1}$ which is transmitted to $v_{i-1}$. by $v_{i-1}$.

$W_{v_{i-1}}\left(t_{i-1}\right) \leq \beta\left(v_{i-1}\right)$, delay $_{v_{i-1} \rightarrow v_{i}}\left(t_{i-1}\right)=d_{\text {edge }}+d_{\text {node }}$

otherwise

delay $_{v_{i-1} \rightarrow v_{i}}\left(t_{i-1}\right)=d_{\text {edge }}+d_{\text {node }} \cdot\left[\frac{W_{v_{i-1}}\left(t_{i-1}\right)}{\beta\left(v_{i-1}\right)}\right]^{\delta}$

Where, $d_{\text {edge }}$ $d_{\text {node }}$ and $\delta$ are constants more than zero. $d_{\text {node }}$ is the consumed time of reading data once of sensor node from $m_{b \text {; }} d_{e d g e}$ is the consumed time of data transmission between adjacent nodes. $\delta$ is the queuing parameters.

Under constraint of time delay, the reliability of both ends of $3 s-W S N$ is defined as: for the provided tristate wireless sensor networks 3 s- $W S N=<V, E, M, Q, S, v_{\text {sink }}>$, selecting any $v_{S} \in V$ as the source node, $v_{t}=v_{\text {sink }}$ is the sink node of the network. The reliability of both ends of WSN is the probability of there being at least one an efficient data transmission link from $v_{s}$ to $v_{t}$. It is recorded as $\operatorname{Rel}_{3 s-W S N}\left(v_{s}, v_{t}\right)$, namely

$$
\begin{aligned}
& \operatorname{Rel}_{3 s-W S N}\left(v_{s}, v_{t}\right)= \\
& \operatorname{Pr}\left(\exists r \operatorname{Path}^{v_{s} \rightarrow v_{t}}\left(\sum_{i=1}^{n} \operatorname{delay~}_{v_{i-1} \rightarrow v_{i}}\left(t_{i-1}\right) \leq T\right)\right)
\end{aligned}
$$

Where, $\mathrm{n}$ is the length of $r \mathrm{Path}^{v_{\mathrm{s}} \rightarrow v_{t}}$.

\section{Reliability of tristate WSN under the effect of IPC and CCF}

Assumed that only IPC phenomenon can occur independently for $m_{c o}$ in node of 3s-WSN. The $m_{c o}$ of each node may have three mutually exclusive events: NF ( $m_{c}$ effective), CF ( $m_{c}$ failure and failure being covered) and SF ( $m_{c}$ failure and failure not being covered). For any node $v \in V$, the probability of occurrence of the above events of $m_{c o}$ are respectively: $p_{N F}(v)=1-q_{c o}(v)+r(v) \cdot q_{c o}(v)$; $p_{C F}(v)=c(v)+q_{c o}(v) ; p_{S F}(v)=s(v)+q_{c o}(v)$, of which, $r(v), c(v)$ and $s(v)$ are called as covering factors and $r(v)+c(v)+s(v)=1$.

Under the common influence of CCF and IPC, the solution of reliability of 3s-WSN must separate the existence conditions of CCF and IPC from the feasible combination of $r \mathrm{Path}^{v_{s} \rightarrow u_{t}}$. 
Under the influence of IPC, node state space is adjusted to $S=\left\{c s, c, f_{c}, f_{s}\right\}$. FC status indicates $m_{c o}$ failure and the failure is covered by WSN detection. $f_{s}$ status indicates $m_{c o}$ failure and the failure is not covered.

Under the influence of IPC, reliability of 3s-WSN is:

$$
\begin{aligned}
\operatorname{Rel}_{3 s-W S N}\left(v_{s}, v_{t}\right)^{I P C} & =P_{\mathrm{Rel}}^{E_{0}} \\
& =P_{\mathrm{Rel}}^{E_{1}} \cdot \operatorname{Pr}\left(E_{1}\right)+P_{\mathrm{Rel}}^{E_{2}} \cdot \operatorname{Pr}\left(E_{2}\right) \\
& =P_{c} \times P_{\text {Rel }}^{E_{2}}
\end{aligned}
$$

Where $P_{c}=\operatorname{Pr}\left(E_{2}\right)=\prod_{v \in V}\left(1-p_{f_{s}}(v)\right), E_{1}$ and $E_{2}$ are two mutually-exclusive integrated events. $E_{1}$ represents that there is at least a node in $f_{s}$ state in 3s-WSN. $E_{2}$ represents that there is no node in $f_{s}$ state in 3s-WSN. $E_{0}=E_{1} \cup E_{2}$. Specify that $P_{\mathrm{Re} l}^{E_{i}}$ is probability of 3s-WSN to meet reliability definition under 3s-WSN condition. Adjust state probability space of each node $\mathrm{v}$ to conditional probability under situation $E_{2}$ occurring before calculating $P_{\mathrm{Rel}}^{E_{2}}$. i.e. $P_{c s}^{E_{2}}(v)=p_{c s}(v) /\left(1-p_{f_{s}}(v)\right)$; $P_{c}^{E_{2}}(v)=p_{c}(v) /\left(1-p_{f_{s}}(v)\right) ; P_{f_{c}}^{E_{2}}(v)=p_{f_{c}}(v) /\left(1-p_{f_{s}}(v)\right)$.

After isolating existence conditions of IPC, we consider CCF in the next. Each CC (common cause) can be regarded as a basic common cause event. There are three relationships between basic common cause events in WSN, i.e. mutual exclusion, mutual independence and correlative dependence. $\mathrm{M}$ CC can constitute $2^{\mathrm{m}}$ mutually-exclusive common cause events (CCE): $C C E_{1}=\overline{C C_{1}} \cap \overline{C C_{2}} \cap \ldots \cap \overline{C C_{m}}, C C E_{2}=C C_{1} \cap \overline{C C_{2}} \cap \ldots \cap \overline{C C_{m}}, \ldots, C C E_{2^{m}}=C C_{1} \cap C C_{2} \cap \ldots \cap C C_{\mathrm{m} . .}$ $\mathrm{P}\left(C C E_{\mathrm{j}}\right)$ represents probability of $C C E_{\mathrm{j}}$ to occur, and $\sum_{j=1}^{2^{m}} P\left(C C E_{j}\right)=1$. Integrate CCF to numeration of $P_{\mathrm{Rel}}^{E_{2}}$ :

$$
\begin{gathered}
P_{R e l}^{E_{2}}(\mathrm{CCF})=\sum_{j=1}^{2^{m}}\left[P_{R e l}^{E_{2}} \mid C C E_{j}\right] \cdot P\left[C C E_{j}\right] \\
P_{\text {Rel }}^{E_{2}} \mid C C E_{j}=
\end{gathered}
$$

Where, $\operatorname{Pr}\left\{\exists r \operatorname{Path}^{v_{s} \rightarrow v_{i}}\left(\sum_{i=1}^{n} \operatorname{delay}_{v_{i-1} \rightarrow v_{i}}\left(t_{i-1}\right) \leq T\right) \mid\left(\left(v_{i-1}, v_{i} \notin A_{C C E_{j}}\right) \cap E_{2}\right)\right\}, \quad A_{C C E_{j}}$ represents nodes affected by CCE $E_{\mathrm{j}}$.

Nodes in all $\left\{v \mid v \in A_{C C E_{j}}\right\}$ are considered invalid when performing calculation. Each state probability of effective node in $r P a t h^{v_{s} \rightarrow v_{t}}$ is applied to calculation of reliability of 3s-WSN under $C C E_{\mathrm{j}}$ after it is adjusted. Existence conditions of CCF and IPC are isolated from WSN model. IPC and CCF together affect calculation of reliability of $3 s-W S N$, thus being simplified.

\section{Experiment and Result Analysis}

This paper performs analysis to impact of IPC and CCF on WSN and performance characteristic of WSN node by integrating them in one model, providing more comprehensive information for reliability analysis of real network system. Next, compare model constructed in this paper with ordinary WSN model (ignore processing delay of node data and impact of IPC, CCF). Algorithm in this paper is based on Meddly-0.7 MDD function library developed by Iowa State University, realizing program running in CentOS-5.5. Kernel version is 2.6.18.

Choose Fig.1 (WSN figure where node number is 64 and link number is 70) as sample. Set node $q_{c o}=0.2, q_{s}=0.5$ (original node $q_{c o}=0, q_{s}=0$ ). Sensing node $g(v)$ is 5.0 packet $/ \mu \mathrm{s}, \beta(v)$ is 20.0 packet/ $\mu$ s and $d_{\text {edge }}$ is $5.0 \mu \mathrm{s}$, and $d_{\text {node }}$ is $1.0 \mu \mathrm{s}$ in 3s-WSN model. Value of $\delta$ is 1 , and covering factor $\mathrm{r}=0, \mathrm{c}=0.97, \mathrm{~s}=0.03$. Set common cause set $C C=\left\{C C_{1}, C C_{2}\right\}$ in WSN, and $C C_{1}$ represents earthquake and $C C_{2}$ represents flood. $C C G_{1}=\left\{v_{31}\right\}, C C G_{2}=\left\{v_{50}, v_{57}\right\}$ (Nodes are ordered from right to left and from up to down), and $P_{C C_{1}}=0.02, \operatorname{Pr}\left(C C_{2} \mid C C_{1}\right)=p_{h}=0.6, \operatorname{Pr}\left(C C_{2} \mid \overline{C C}_{1}\right)=p_{\bar{h}}=0.03$. In ordinary model which ignores processing delay of sensing node data and impact of IPC, CCF, delay value (only transmits to directed link) $d_{e d g e}=1.0 \mu \mathrm{s}$, and ignore IPC and CCF. With increasing of delay constraint T, reliability change situation of WSN under the background of two models is as shown in Fig. 2. 


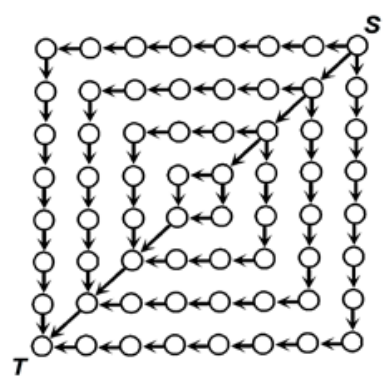

Fig.1. 64 nodes WSN

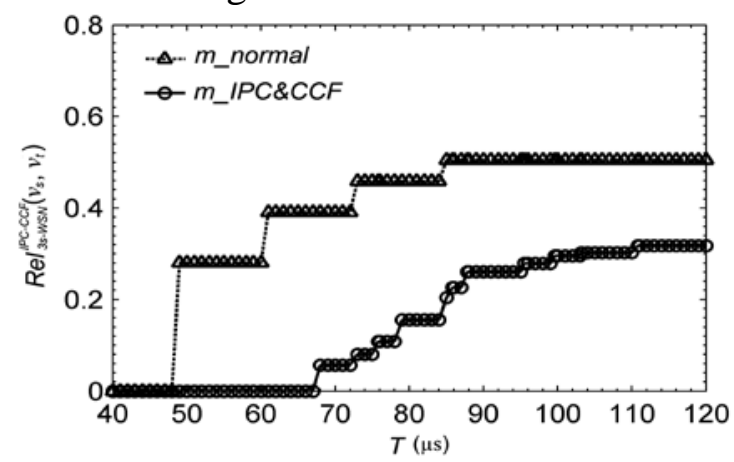

Fig.2. Comparison of m_IPC\&CCF and m_nomal Model

Consider processing delay of node data and impact of IPC and CCF at the same time. Change of $\operatorname{Rel}_{3 s-W S N}\left(v_{s}, v_{t}\right)$ conforms to change of Delay constraint value, and we can see it by observing curve painted with circles in figure (recorded as m_IPC\&CCF). When only link transmission delay is considered and impact of IPC and CCF is ignored, reliability change of WSN calculated is as shown in curve painted with three angles (recorded as m_nomal). From Fig.6, we can see that under the situation that only link transmission delay of WSN is considered and impact of IPC and CCF is ignored, the calculated reliability of WSN is obviously higher than that of WSN. For example, when $T=68.0 \mu \mathrm{s}$, and $\mathrm{m}_{-}$IPC\&CCF, $\operatorname{Rel}_{3 \mathrm{~s}-W S N}\left(v_{s}, v_{t}\right)$ of model $=0.0569292$, but the calculated reliability of WSN under m_nomal model is 0.391057 . Therefore, in reliability assessment of multistate WSN, node data queueing delay due to network congestion and impact of IPC and CCF on network reliability cannot be ignored.

\section{Conclusion}

CCF analysis involves considering failure of many system components at the same time. Difficulty of introducing lies in the processing of many failure modes of component. This paper firstly expands IPC model ${ }^{[5]}$ put forward by Dugan to multistate WSN where internal module state of node is independent, and considers IPC and CCF, performing analysis to reliability of 3-states WSN under delay constraint. Integrate node performance and failure performance of WSN in one model to discuss it. Based on symbol algorithm of reliability given by MMDD, reliability of WSN can be gained by establishing one MMDD (according to common cause and MMDD node generated by traversal). It can not only accurately calculate reliability of 3-states WSN under impact of IPC and CCF, but also has higher space storage efficiency.

\section{References}

[1] Yang J, Zhou J, Lv Z, et al. A Real-Time Monitoring System of Industry Carbon Monoxide Based on Wireless Sensor Networks[J]. Sensors, 2015, 15(11): 29535-29546.

[2] Jiang D, Ying X, Han Y, et al. Collaborative multi-hop routing in cognitive wireless networks[J]. Wireless Personal Communications, 2016, 86(2): 901-923. 
[3] Lv Z, Halawani A, Feng S, et al. Multimodal hand and foot gesture interaction for handheld devices[J]. ACM Transactions on Multimedia Computing, Communications, and Applications (TOMM), 2014, 11(1s)

[4] Jinyu Hu and Zhiwei Gao. Distinction immune genes of hepatitis-induced heptatocellular carcinoma[J]. Bioinformatics, 2012, 28(24): 3191-3194. 